\title{
Antagonizing reactive oxygen by treatment with a manganese (III) metalloporphyrin-based superoxide dismutase mimetic in cardiac transplants
}

Vani Nilakantan, $\mathrm{PhD},{ }^{\mathrm{a}}$ Xianghua Zhou, MD, ${ }^{\mathrm{a}}$ Gail Hilton, MS, ${ }^{\mathrm{a}}$ Yang Shi, PhD, ${ }^{\mathrm{b}}$ John E. Baker, PhD, Ashwani K. Khanna, $\mathrm{PhD}^{\mathrm{c}}$ and Galen M. Pieper, $\mathrm{PhD}^{\mathrm{a}, \mathrm{d}}$

From the Department of Surgery, Divisions of Transplant Surgery a and Pediatric Surgery, ${ }^{\mathrm{b}}$ the Department of Medicine, ${ }^{\mathrm{c}}$ and the Free Radical Research Center, ${ }^{\mathrm{d}}$ Medical College of Wisconsin, Milwaukee, Wis.

Supported, in part, by in-kind support from the VA Medical Center, Milwaukee, Wis, and by National Institutes of Health grants HL64637 (G.M.P.) and HL54075, HL66334, HL65203 (J.E.B.) and by American Heart Association grant 03653822 (Y.S.).

Received for publication July 28, 2005; revisions received Oct 14, 2005; accepted for publication Nov 8, 2005.

Address for reprints: Galen M. Pieper, PhD, Transplant Surgery, Medical College of Wisconsin, 9200 West Wisconsin Ave, Milwaukee, WI 53226 (E-mail: gmpieper@ mcw.edu)

J Thorac Cardiovasc Surg 2006;131:898-906 $0022-5223 / \$ 32.00$

Copyright $\odot 2006$ by The American Association for Thoracic Surgery

doi:10.1016/j.jtcvs.2005.11.015
Objective: Oxidative stress might be an important factor contributing to injury during alloimmune activation. Herein, we evaluated the efficacy of a superoxide dismutase mimetic, manganese (III) tetrakis (1-methyl-4-pyridyl) porphyrin pentachloride (MnTmPyP), on cytokine gene expression and apoptotic signaling in a rat model of cardiac transplantation.

Methods: Lewis $\rightarrow$ Lewis (isografts) or Wistar-Furth $\rightarrow$ Lewis (allografts) heterotopic rat transplants without and with treatment with MnTmPyP were used. Reactive oxygen formation was determined on the basis of dihydroethidine fluorescence and lucigenin-enhanced chemiluminescence. In situ graft function was determined by means of sonomicrometry. Inflammatory cytokine, proapoptotic, and antiapoptotic gene expression at either postoperative day 4 (early rejection) or postoperative day 6 (late rejection) was determined by means of reverse transcriptase polymerase chain reaction.

Results: An increased production of reactive oxygen in allografts was inhibited to isograft control levels by MnTmPyP. MnTmPyP restored either the percentage of fractional shortening, the distended diastolic and systolic myocardial segment lengths, or both in allografts. Of the increases in cytokine and proapoptotic gene expression in allografts, only interleukin 6 was decreased by MnTmPyP. MnTmPyP inhibited antiapoptotic gene expression (Bcl-2 and $\mathrm{Bcl}-\mathrm{xL}$ ) during early rejection but restored expression at later stages. The increase in activated caspase-3 levels in allografts was inhibited by MnTmPyP.

Conclusions: The mechanism of the beneficial effect of MnTmPyP on graft function appear related, in part, to scavenging $\mathrm{O}_{2}^{--}$and by decreasing apoptotic signaling rather than an effect on inflammatory cytokine gene expression.

$\mathrm{O}$ rgan transplant rejection, including cardiac allograft rejection, is associated with inflammation and alloimmune activation. It is generally acknowledged that this is associated with enhanced oxidative stress, although there has been no direct determination of reactive oxygen species production in cardiac allograft rejection. Thus counteracting the effects of oxidative stress might prove to be an important mechanism of protecting tissue grafts during the inflammatory response in cardiac transplants. In this context we have determined that diverse antioxidants, such as ascorbic acid, dimethylthiourea, or pyrrolidine dithiocarbamate, limit rejection, prolong graft survival, or both. ${ }^{1-3}$ These data suggest that reactive oxygen species play a role in acute cardiac rejection.

Previously, we reported that total superoxide dismutase (SOD) content in rat cardiac transplants was decreased before graft failure, ${ }^{4}$ confirming observations that the loss in antioxidant enzymes might be an important contributor to 


$$
\begin{aligned}
& \text { Abbreviations and Acronyms } \\
& \text { FasL } \quad=\text { Fas ligand } \\
& \text { IFN- } \gamma=\text { interferon } \gamma \\
& \text { IL } \quad=\text { interleukin } \\
& \text { iNOS = inducible nitric oxide synthase } \\
& \text { MnSOD = manganese superoxide dismutase } \\
& \text { MnTBAP = Manganese (III) tetrakis(4-benzoic acid) } \\
& \text { porphyrin chloride } \\
& \text { MnTmPyP = Manganese (III) tetrakis(1-methyl-4- } \\
& \text { pyridyl) porphyrin pentachloride } \\
& \text { NO }=\text { nitric oxide } \\
& \mathrm{POD}=\text { postoperative day } \\
& \text { RT-PCR = reverse transcriptase polymerase chain } \\
& \text { reaction } \\
& \text { SOD }=\text { superoxide dismutase } \\
& \mathrm{TNF}-\alpha=\text { tumor necrosis factor } \alpha
\end{aligned}
$$

tory cytokine and proapoptotic and antiapoptotic gene expression.

\section{Methods \\ Transplantation and Treatment}

All animal procedures were approved by the local institutional animal care and use committee. All animals received humane care in compliance with the "Guide for the Care and Use of Laboratory Animals." Lewis (Lew:RT1 ${ }^{1}$ ) and Wistar-Furth (WF:RT1 ${ }^{\mathrm{u}}$ ) rat strains were chosen for genetic disparity at both the major and minor histocompatibility loci for donor-to-recipient combination of Lewis $\rightarrow$ Lewis (for isografts) or Wistar-Furth $\rightarrow$ Lewis (for allografts) rats. Isogeneic and allogeneic heterotopic cardiac transplantation was performed under sterile conditions, as previously described. ${ }^{4}$ Donor hearts were arrested in cold University of Wisconsin preservation solution and transplanted to recipients. Total cold ischemic time was less than 35 minutes for all experimental groups. To test the principle of action of MnTmPyP on $\mathrm{O}_{2}{ }^{--}$production, some allograft recipients received daily intraperitoneal injections of $10 \mathrm{mg} / \mathrm{kg}$ MnTmPyP beginning the day of the operation after transplantation and continuing until postoperative day (POD) 4 or 6 . This is a dose that has been used primarily in rodent models of acute inflammation by using a single-injection strategy. In subsequent studies we also evaluated a lower dose of $5 \mathrm{mg} / \mathrm{kg}$ in some of the allograft recipients to determine a dose response on measures of graft function and cytokine and apoptotic gene expression. During the experimental period, there was no acute loss of grafts in any group. At POD4 and POD6, grafts were arrested and flushed with cold University of Wisconsin solution and minced, and portions were either frozen in liquid $\mathrm{N}_{2}$ and stored at $-80^{\circ} \mathrm{C}$ for reverse transcriptase polymerase chain reaction (RT-PCR) and caspase-3 activity assays or used immediately for assay of reactive oxygen production by using lucigeninenhanced chemiluminescence assays.

\section{Graft Function}

Graft function was quantitated in situ by using sonomicrometry (Sonometrics Corp, London, Ontario, Canada) before arrest and harvesting of tissue for various analyses (described below). In preliminary studies (unpublished) we found that there were no significant differences in heart rates, segment lengths, or percentages of segment shortening between isografts and allografts through POD4, thereby eliminating any possible differences in function caused by rat strains. Thus all analyses for this study were performed at POD6 for each group $(n=6$ each). Recipient rats were anesthetized with pentobarbital $(50 \mathrm{mg} / \mathrm{kg}$ administered intraperitoneally), and 2 piezoelectric crystals were placed at the midheart level to determine short-axis dimension-graft function, as previously reported. ${ }^{14}$

\section{Detection of Reactive Oxygen Species by Using Dihydroethidine Fluorescence}

Heparin (150 U/kg administered intraperitoneally) was administered to animals ( $\mathrm{n}=4$ each), followed by induction of anesthesia with sodium pentobarbital $(50 \mathrm{mg} / \mathrm{kg}$ administered intraperitoneally). Transplanted hearts were removed and placed in $4^{\circ} \mathrm{C}$ Krebs-Henseleit buffer, and the aorta was cannulated for ex vivo perfusion. Hearts were perfused retrochloride (MnTmPyP) provided protection in acute cardiac allograft rejection. We determined efficacy on the basis of measures of graft function, as well as inflamma- 
gradely with bicarbonate buffer at a constant pressure for 10 minutes to wash out blood and then perfused for 20 minutes with buffer containing $10 \mu \mathrm{mol} / \mathrm{L}$ hydroethidine to detect reactive oxygen species production, as previously described in our laboratories. ${ }^{15}$ Because of this intervention, these hearts could not be used for biochemical and RT-PCR analysis.

At the end of the perfusion, hearts were frozen in OCT 4583 and sectioned. Frozen sections $10 \mu \mathrm{m}$ thick were cut and thaw mounted on slides. A cover slip was applied to the sections on the slides, and images were obtained with a digital camera and a Nikon E600 microscope equipped with epifluorescence (excitation, $488 \mathrm{~nm}$; emission, $610 \mathrm{~nm}$ ). The fluorescence intensity of nuclei in 40 cells from each section was measured and was corrected for background fluorescence in nonnuclear regions with MetaMorph software (Visitron Systems GmbH, Puchheim, Germany). Four sections per heart and 3 hearts per group were studied. Animals were administered either MnTmPyP or manganese (III) tetrakis (4-benzoic acid) porphyrin chloride MnTBAP 1 hour before the hydroethidine staining.

\section{Reactive Oxygen Species Detection by Using Lucigenin-enhanced Chemiluminescence}

Approximately $100 \mathrm{mg}$ of heart tissue from isografts and allografts $(\mathrm{n}=6$ each) was homogenized in $10 \mathrm{~mL}$ of Krebs-Henseleit buffer containing $10 \mathrm{mmol} / \mathrm{L} \mathrm{N}$-2-hydroxyethylpiperazine- $\mathrm{N}$-2ethanesulfonic acid (HEPES) similar to that previously described for cardiac tissue. ${ }^{16}$ The tubes containing $1 \mathrm{~mL}$ of the homogenate or buffer as blank were incubated in the dark either alone or with $100 \mu \mathrm{mol} / \mathrm{L}$ MnTmPyP for 30 minutes. Lucigenin-enhanced chemiluminescence was then measured for 3 minutes in a luminometer (Berthold Instruments; Autolomat LB 953, Postfach, Germany) after the addition of $20 \mu \mathrm{mol} / \mathrm{L}$ lucigenin to each tube. The tube containing the buffer and lucigenin was read first to calculate background luminescence, and this value was subtracted from each subsequent value. A BioRad (Hercules, Calif) protein assay was performed for assessment of protein in milligrams per milliliter. Reactive oxygen production was reported as relative light units emitted per milligram of protein.

\section{Plasma Nitric Oxide Metabolites}

The nitric oxide (NO) byproducts, nitrate and nitrite, were measured ( $\mathrm{n}=6$ each) by the Griess reaction after conversion of nitrate to nitrite by using nitrate reductase with a commercial kit (Cayman Chemical, Ann Arbor, Mich).

\section{Inflammatory Cytokine and Apoptotic Gene Expression}

Total RNA was purified by using the Promega SV Total RNA Isolation System (Promega, Madison, Wis), according to the manufacturer's directions. RNA concentration was determined spectrophotometrically. cDNA was synthesized from $1 \mu \mathrm{g}$ of total RNA and oligo(dT) primers by using the Invitrogen Superscript First-Strand Synthesis System for RT-PCR (Invitrogen, Carlsbad, Calif), according to the manufacturer's directions. One microliter of cDNA was mixed with 25 pmol of specific sense and antisense primers and Invitrogen PCR Supermix to a volume of $25 \mu \mathrm{L}$, and the reaction was incubated in a BioRad iCycler (BioRad) under the following conditions: for inducible nitric oxide synthase (iNOS) and $\mathrm{Bcl}-2,94^{\circ} \mathrm{C}(60$ seconds), $60^{\circ} \mathrm{C}$ (60 seconds), and $72^{\circ} \mathrm{C}$ (60 seconds) for 30 cycles; for interferon $\gamma$ (IFN- $\gamma$ ), interleukin (IL) 6, IL-10, and tumor necrosis factor $\alpha$ (TNF- $\alpha), 95^{\circ} \mathrm{C}$ (30 seconds), $60^{\circ} \mathrm{C}(30$ seconds), and $72^{\circ} \mathrm{C}$ (60 seconds) for 35 cycles; for $\mathrm{Bcl}-\mathrm{xL}$, $95^{\circ} \mathrm{C}$ (30 seconds), $60^{\circ} \mathrm{C}$ (30 seconds), and $72^{\circ} \mathrm{C}$ (60 seconds) for 37 cycles; and for Fas ligand (FasL), $95^{\circ} \mathrm{C}$ (60 seconds), $58^{\circ} \mathrm{C}$ (60 seconds), and $72^{\circ} \mathrm{C}(60$ seconds $)$ for 31 cycles.

Ten microliters of the PCR product was resolved by $1 \%$ agarose gel electrophoresis. Ethidium bromide-stained specific bands were visualized under ultraviolet light, and densitometric analysis of specific bands were made with the Alpha Imager (Alpha Innotech Corp, San Leandro, Calif) and expressed as a ratio to $\beta$-actin gene controls.

\section{Caspase Activity}

Caspase-3 activity assay ( $\mathrm{n}=4-6$ each group) was performed with a commercial kit purchased from Sigma-Aldrich (St Louis, Mo). This assay is based on the hydrolysis of the peptide substrate acetyl-ASP-Glu-Val-Asp-p-nitroanilide by caspase-3 to release $p$ nitroaniline, which is measured spectrophotometrically at $405 \mathrm{~nm}$ on a 96-well enzyme-linked immunosorbent assay reader. Caspase enzyme activity was determined in units of micromoles of $p$ nitroaniline per minute per milliliter.

\section{Data Analysis}

All values are expressed as means \pm standard error of the mean. Statistical analysis was performed with 1-way analysis of variance with the Student-Newman-Keuls test for multiple comparisons of group means or with the Student $t$ test for comparisons between 2 group means. Analysis was performed with GraphPad InStat software (San Diego, Calif).

\section{Results}

\section{Reactive Oxygen Species Production}

We used 2 methods to determine reactive oxygen species production: dihydroethidine fluorescence microscopy and lucigenin-enhanced chemiluminescence. For the former, these studies were performed at POD4 because poor perfusion caused by rejection at POD6 limited optimal perfusion of dye to make this reliable at this time period. We found increases $(P<.05)$ in fluorescence intensity in cardiac allografts compared with isografts perfused with dihydroethidine, suggesting enhanced reactive oxygen species production (Figure 1). Similar to pilot studies with the more traditional probe MnTBAP, we found that fluorescence was decreased $(P<.01)$ to background isograft levels in allograft recipients injected in vivo with MnTmPyP.

In addition, for quantitative purposes, we performed lucigenin-enhanced chemiluminescence from cardiac homogenates derived from grafts at POD6. We found enhanced chemiluminescence in cardiac allografts compared with isograft controls (Figure 2). To document that this chemiluminescence technique was specific for $\mathrm{O}_{2}{ }^{--}$production, we show that the chemiluminescence signal in both 

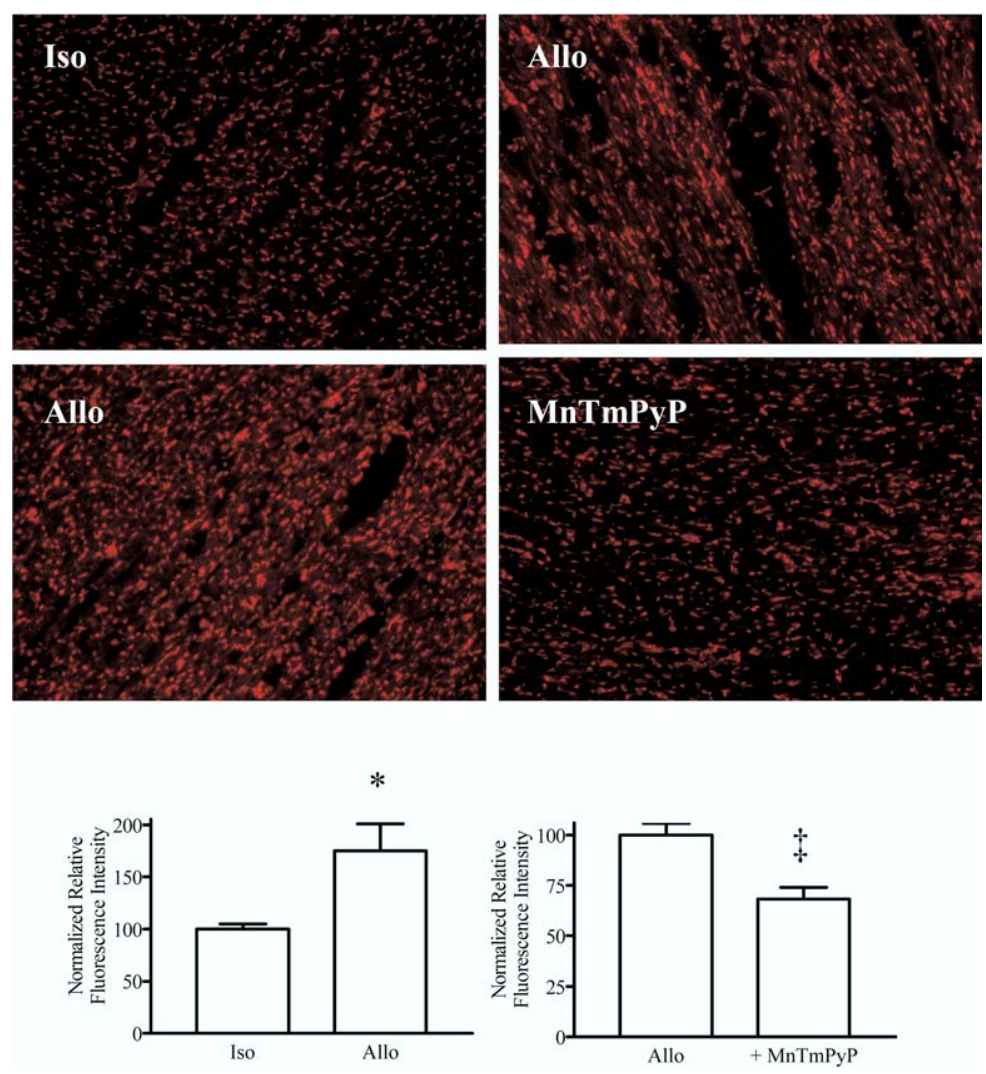

Figure 1. Effect of MnTmPyP on fluorescence in transplanted hearts perfused ex vivo at postoperative day 4 with dihydroethidine. Quantification of pair-matched reactive oxygen production in isografts (Iso) versus untreated allografts $(A / / 0)$ and allografts treated daily with $\operatorname{MnTmPyP}\left(\mathrm{n}=4\right.$ each). ${ }^{*} P<.05 . \ddagger P<.01$.

isografts and allografts was abolished by MnTmPyP. To show the effect of scavenging $\mathrm{O}_{2}{ }^{--}$on NO bioactivity, we measured plasma NO metabolites ( $n=6$ each). We found that MnTmPyP increased plasma NO metabolites versus untreated allografts (plasma NO: isografts, $13.94 \pm 2.19$

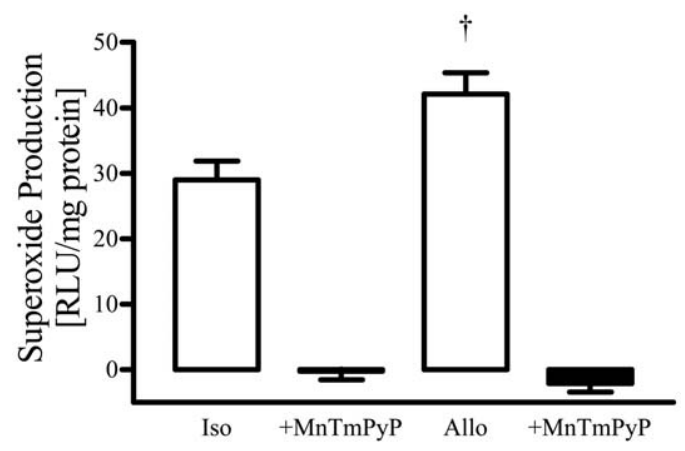

Figure 2. Increased lucigenin-enhanced $\mathrm{O}_{2}^{--}$production in cardiac homogenates from allografts $(\mathrm{A} / \mathrm{lo})$ harvested at postoperative day 6 versus isografts (Iso) and blockade by MnTmPyP (n = 6 each). $+P<.025$. $\mu \mathrm{mol} / \mathrm{L}$; allografts, $38.19 \pm 3.06 \mu \mathrm{mol} / \mathrm{L}$; MnTmPyPtreated allografts, $49.73 \pm 2.15 \mu \mathrm{mol} / \mathrm{L} ; P<.05$, treated vs untreated). This change occurred independent of any changes in iNOS gene expression normalized to $\beta$-actin (gene ratio in arbitrary densitometry units: isografts, 0.038 \pm 0.021 ; allografts, $0.508 \pm 0.406$; MnTmPyP-treated allografts, $0.499 \pm 0.090)$. Collectively, these data indicated that the increased chemiluminescence arises through $\mathrm{O}_{2}{ }^{--}$ production and that MnTmPyP acts to restore NO bioactivity by decreasing $\mathrm{O}_{2}{ }^{--}$levels in cardiac tissue.

\section{Graft Function}

Using in situ sonomicrometry in grafts before harvest on POD6, we noted decreases $(P<.01)$ in heart rate of untreated allografts versus isografts (Figure 3 ). Furthermore, both end-diastolic and end-systolic segment lengths were increased $(P<.001)$, whereas the percentage of fractional shortening was decreased $(P<.001)$ in allografts compared with isografts. Treatment with both doses of MnTmPyP normalized both end-diastolic and end-systolic lengths to isograft control levels. The lower dose of MnTmPyP also 

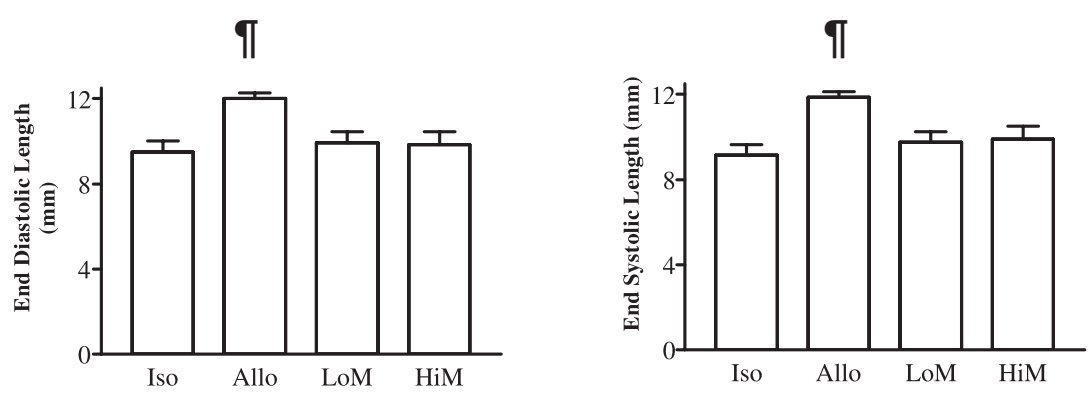

Figure 3. Effect of MnTmPyP on in situ graft function determined by means of transverseaxis sonomicrometry measured on postoperative day 6 ( $n=6$ each). $\Uparrow P<.001$ and $¥ P<$ .01 vs allografts. Iso, Isografts; Allo, allografts; LoM, low-dose MnTmPyP; HiM, highdose MnTmPyP.
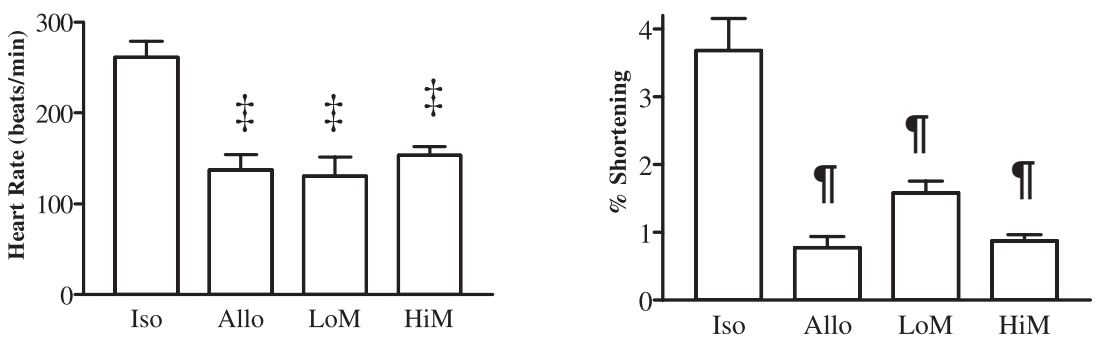

partially improved the percentage of fractional shortening $(P<.001$ vs untreated allografts $)$.

Inflammatory Cytokine and Proapoptotic and Antiapoptotic Gene Expression

In samples harvested at POD4 and POD6, we found significant increases in inflammatory cytokine gene expression for IFN- $\gamma$ and the $\mathrm{T}_{\mathrm{H}} 2$ cytokines IL-6 and IL-10 (Figures 4 and 5). Treatment with MnTmPyP caused a decrease in IL-6 but not IL-10 or INF- $\gamma$ at POD4. Later, at POD6, MnTmPyP did not alter any cytokine expression (Figures 4 and 5).

Apoptosis in rejecting cardiac allografts could be influenced by changes in either proapoptotic or antiapoptotic gene expres-

\section{POD4}
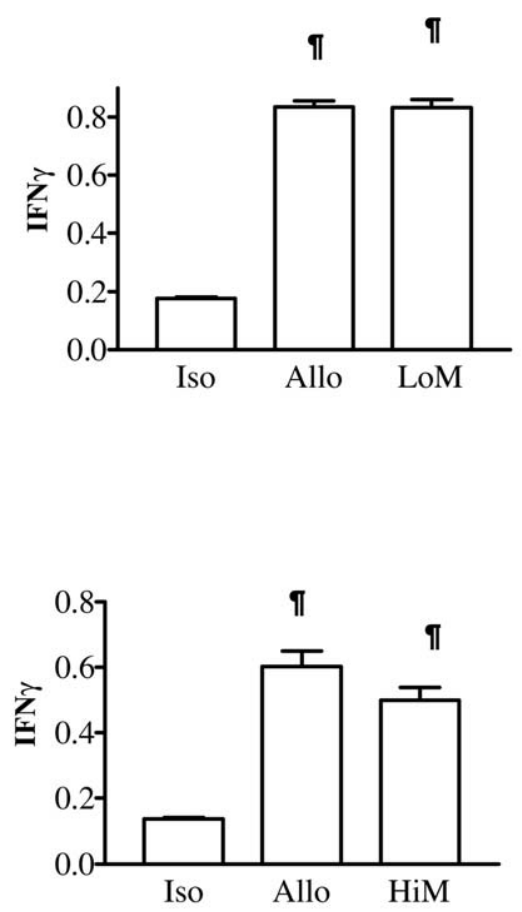

POD6
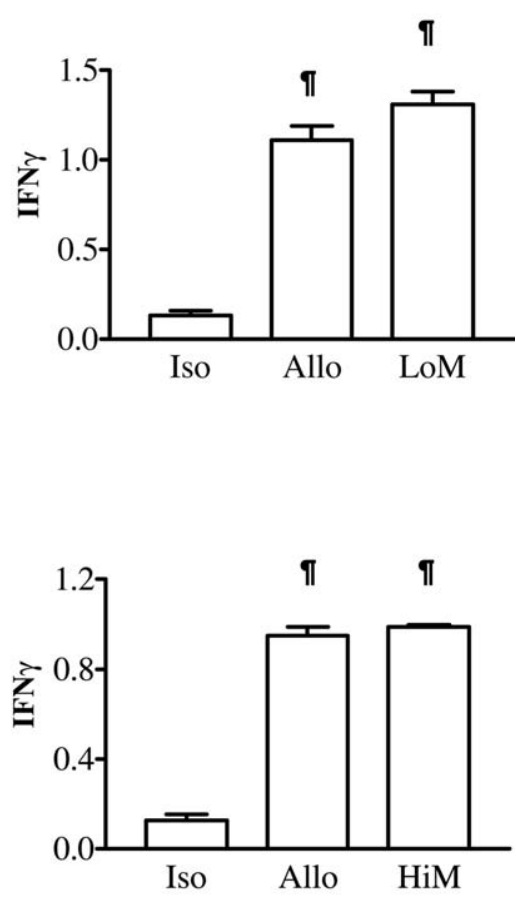

Figure 4. Densitometry of reverse transcriptase polymerase chain reaction for interferon $\gamma(I F N \gamma)$ gene expressed as a ratio to the housekeeping gene $\beta$-actin, showing the effect of low- and high-dose MnTmPyP (LoM and $H i M)$ gene expression at postoperative days (POD) 4 and 6 (isografts [Iso], $\mathrm{n}=3$ each; allografts [AIlo] without and with treatment, $\mathrm{n}=4$ each). $\Pi P<.001$ versus isografts. 


\section{$\underline{\text { IL-6 }}$}

POD4
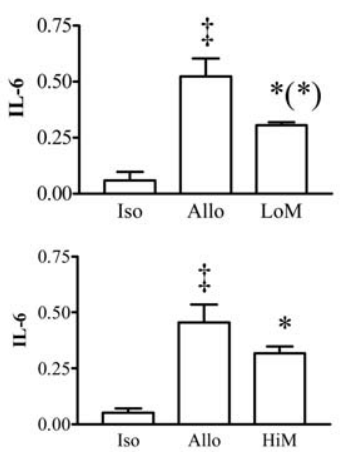

POD6
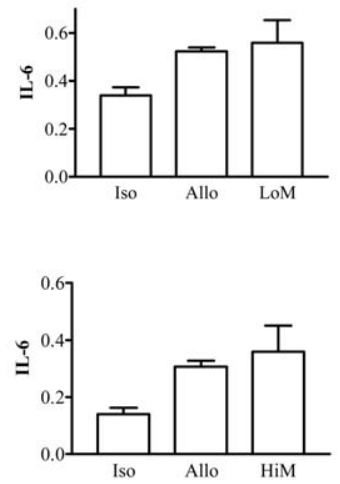

$\underline{\text { IL-10 }}$
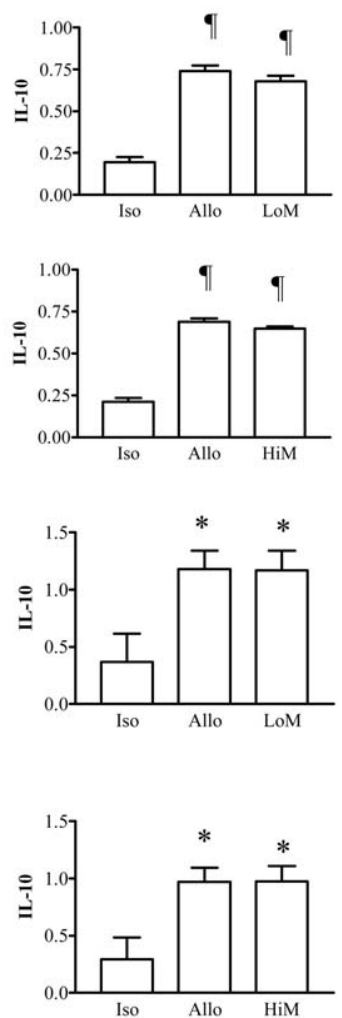

Figure 5. Densitometry of reverse transcriptase polymerase chain reaction for $\mathrm{T}_{\mathrm{H}} 2$ (interleukin [IL] 6 and interleukin 10) cytokine genes expressed as a ratio to the housekeeping gene $\beta$-actin, showing the effect of MnTmPyP on gene expression at postoperative days (POD) 4 and 6 (isografts [Iso], $\mathrm{n}=3$ each; allografts [AIlo] without and with treatment, $n=4$ each). ${ }^{*} P<.05, \pm P<.01$, and $\Pi P<.001$ versus isografts; $\left({ }^{*}\right) P<.05$ versus allografts. $L O M$, Low-dose MnTmPyP; HiM, high-dose MnTmPyP.

sion. In untreated allografts, expression of the proapoptotic genes FasL and TNF- $\alpha$ was upregulated at both time points (Figure 6). The antiapoptotic gene $\mathrm{Bcl}-2$ was also upregulated at POD4, although expression of Bcl-xL was unchanged at either time point (Figure 7). MnTmPyP did not modulate FasL or TNF- $\alpha$ expression in cardiac allografts (Figure 6). Surprisingly, the low dose of MnTmPyP also inhibited the expression of the antiapoptotic genes $\mathrm{Bcl}-2$ and $\mathrm{Bcl}-\mathrm{xL}$ only at this time point (Figure 7). At the high dose, MnTmPyP did not alter either Bcl-2 or Bcl-xL gene expression (Figure 7).

\section{Caspase-3 Activation}

We next evaluated caspase- 3 activity to determine that increases in proapoptotic gene expression at early time points resulted in increased apoptotic functional activity. We found that caspase- 3 activity was increased $(P<.05)$ in allografts compared with isograft controls (Figure 8). Treatment with MnTmPyP decreased caspase activity to isograft levels in a dose-dependent manner (Figure 8).

\section{Discussion}

This study shows, for the first time, that continuous treatment with a cell-permeable SOD mimetic, MnTmPyP, has beneficial effects on graft function and apoptotic gene expression in a rat model of acute cardiac transplantation. The major findings are as follows: (1) MnTmPyP was effective in inhibiting reactive oxygen species production in cardiac allografts; (2) the low dose was more effective than the high dose in improving indices of graft function; (3) MnTmPyP partially decreased IL-6 gene expression but not IL-10 or IFN- $\gamma$ expression at the early stages of rejection; (4)

\section{POD4}

$\underline{\text { TNF } \alpha}$
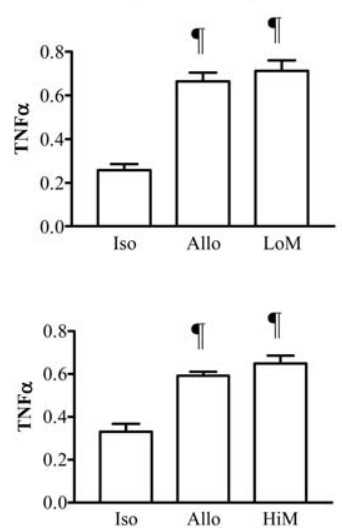

POD6
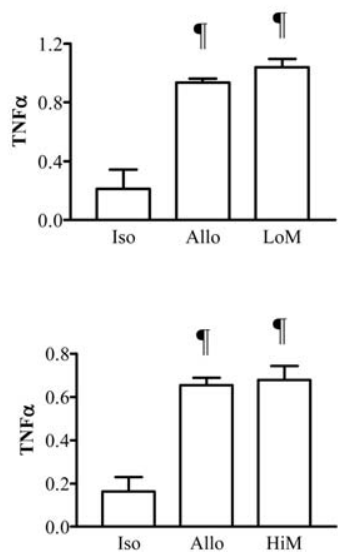

$\underline{\text { FasL }}$
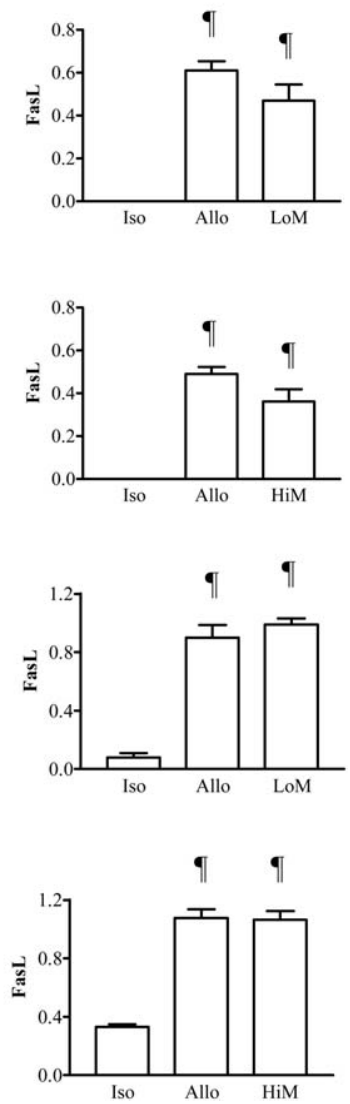

Figure 6. Densitometry of reverse transcriptase polymerase chain reaction for proapoptotic genes expressed as a ratio to the housekeeping gene $\beta$-actin, showing the effect of MnTmPyP on gene expression at postoperative days (POD) 4 and 6 (isografts [Iso], $\mathbf{n}=3$ each; allografts [AIIo] without and with treatment, $\mathrm{n}=4$ each). $\Pi P<.001$ versus isografts. $T N F \alpha$, Tumor necrosis factor $\alpha$; FasL, Fas ligand; LoM, low-dose MnTmPyP; HiM, highdose MnTmPyP. 
MnTmPyP modulated antiapoptotic gene (Bcl-2 and Bcl-xL) expression; and (5) MnTmPyP inhibited caspase-3 activation.

Macrocyclic SOD mimetics have been used with varied results; however, previously reported in vivo studies with MnTmPyP were conducted primarily with a single pretreatment strategy before inducing septic shock and were not used in any multiple-dosing or chronic treatment regimens. In this context our study is the first known application of a continuous daily treatment with a metalloporphyrin on inflammatory and apoptotic gene expression in any transplant model, including cardiac transplantation. MnTmPyP has an advantage over the use of exogenously administered SOD protein in that it is cell permeable. ${ }^{10,17}$ Thus, unlike exogenous SOD protein, MnTmPyP can work effectively to dismutate $\mathrm{O}_{2}{ }^{--}$ intracellularly.

MnTmPyP was effective in blocking reactive oxygen production in allografts, as observed qualitatively by $\underline{\text { Bcl-2 }}$

POD4
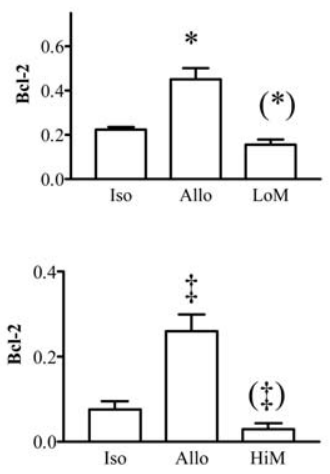

POD6
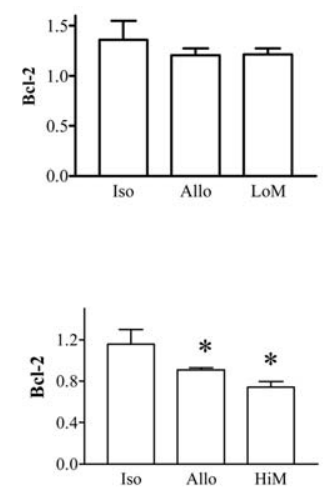

$\underline{\text { Bcl-xL }}$
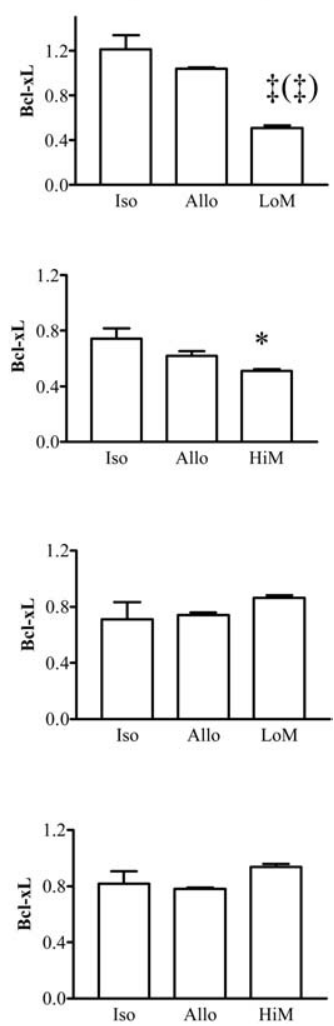

Figure 7. Densitometry of reverse transcriptase polymerase chain reaction for antiapoptotic genes expressed as a ratio to the housekeeping gene $\beta$-actin, showing the effect of MnTmPyP on gene expression at postoperative days (POD) 4 and 6 (isografts [Iso], $\mathbf{n}=\mathbf{3}$ each; allografts [AIlo] without and with treatment, $\mathbf{n}$ $=4$ each). ${ }^{*} P<.05$ and $¥ P<.01$ versus isografts; $\left({ }^{*}\right) P<.05$, $(\ddagger) P<.01$ versus allografts. LoM, Low-dose MnTmPyP; HiM, high-dose MnTmPyP.

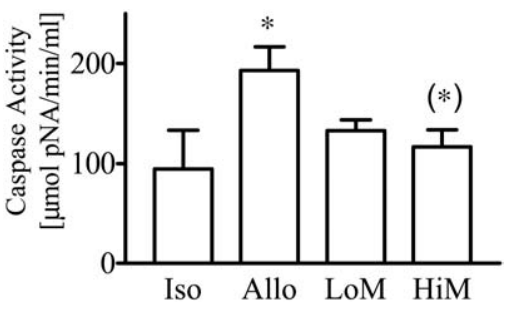

Figure 8. Inhibition by MnTmPyP of alloimmune-induced increase in caspase-3 activity in allografts $(A / / O)$ at postoperative day 6 ( $n=4-6$ each group). ${ }^{*} P<.05$ versus isografts, $\left({ }^{*}\right) P<.05$ vs allografts. Iso, Isografts; LoM, low-dose MnTmPyP; HiM, highdose MnTmPyP.

fluorescence microscopy and quantitatively by lucigeninenhanced chemiluminescence. To our knowledge, this is the first time that either of these 2 techniques has been used to document reactive oxygen production in any experimental or transplant model. Our findings agree with previously published reports indicating that MnT$\mathrm{mPyP}$ effectively inhibits reactive oxygen production in rat cardiomyocytes in response to hypertrophic stimuli. ${ }^{18,19}$ Our studies do not delineate whether there are some other unknown $\mathrm{O}_{2}{ }^{--}$scavenging-independent mechanisms of action of MnTmPyP.

In our study we showed a beneficial action of MnT$\mathrm{mPyP}$ on graft function. Although both doses were effective in reducing the alloimmune-induced increases in end-diastolic and end-systolic lengths, only the low dose was effective in restoring the percentage of segment shortening. This dose-effect property of this nonpeptide SOD mimetic is reminiscent of the decreased efficacy of high doses of exogenous CuZn SOD and MnSOD in ischemia-reperfusion injury in isolated and perfused rabbit or rat hearts. ${ }^{20,21}$ This effect of high-dose SOD protein was not attributed to adverse actions through a peroxidase function of SOD but rather to the fact that that excess scavenging of $\mathrm{O}_{2}^{--}$by the high-dose SOD eliminates the beneficial action of $\mathrm{O}_{2}{ }^{--}$, acting at the step to terminate lipid peroxidation. This biphasic dose dependency of SOD protein can now be applied to in vivo cardiac models by using nonpeptide SOD mimetics.

We have recently reported that MnSOD protein and activity is significantly decreased in cardiac allografts and that this is at least partly due to nitration of MnSOD protein. ${ }^{6}$ Thus, MnTmPyP might have the potential to counteract the loss in this major antioxidant enzyme by improving scavenging of $\mathrm{O}_{2}^{--}$, thus preventing the formation of peroxynitrite, a potent nitrating agent and inducer of apoptosis. In fact, despite a lack of change in iNOS expression, our finding that NO levels are actually increased by $\mathrm{MnTmPyP}$ suggests that $\mathrm{MnTmPyP}$ protects $\mathrm{NO}$ from destruction by $\mathrm{O}_{2}^{\cdot-}$ in allografts. 
Long-term treatment with metalloporphyrinic SOD mimetics might have immunomodulatory activity because treatment with $\mathrm{Mn}(\mathrm{III})$ tetrakis ( $N$-ethylpyridinium-2-yl)porphyrin decreased $\mathrm{T}$ cell-mediated diabetogenesis with decreased inflammatory cell infiltrate in a nontransplant model of alloimmunity. ${ }^{22}$ In our study, treatment with MnTmPyP inhibited IL-6 expression without any effect on IL-10 expression in the early stages but not at the later stages of rejection. High serum IL-6 levels can be predictive of rejection episodes ${ }^{23-25}$; however, it is still not clear whether IL-10 plays a beneficial or a detrimental role in rejection. Recently, in a model of mouse cardiac transplantation, it was shown that hepatocyte growth factor exerts its protective effects by enhancing the expression of IL-10. ${ }^{26}$ The effect of the metalloporphyrinic MnTmPyP in our model is similar to the inability of M40403, a macrocyclic SOD mimetic, to modulate serum IL-6 levels while increasing IL-10 levels in Escherichia coli-mediated septic shock. ${ }^{27}$ Although it is clear that IFN- $\gamma$ plays a significant role in alloimmune activation, the fact that MnTmPyP did not alter either IL-10 or IFN- $\gamma$ expression suggest that MnTmPyP acts downstream of inflammatory cytokine gene expression in our model of cardiac transplantation.

Upregulation of FasL and TNF- $\alpha$ are potential pathways for apoptosis during alloimmune activation in transplanted organs. It has also been shown that $\mathrm{O}_{2}{ }^{-}$is essential for T-cell receptor-stimulated activation of FasL and subsequent cell death. ${ }^{28}$ However, our finding that MnTmPyP did not affect either TNF- $\alpha$ or FasL expression suggests that this metalloporphyrin compound acts distally to FasL or TNF- $\alpha$-mediated apoptosis. This finding is in contrast with a report using another SOD mimetic, MnTBAP, which inhibited Fas-induced liver failure in a mouse model. ${ }^{29}$

Interestingly, at the early stages of rejection (i.e., POD4), MnTmPyP inhibited antiapoptotic gene expression without altering the increased expression of FasL or TNF- $\alpha$. This result was surprising in light of evidence that other metalloporphyrinic SOD mimetics, such as MnTBAP, have been shown to increase Bcl-2 expression in activated T cells. ${ }^{30}$ This action of MnSOD mimetics to oppose apoptosis of activated $\mathrm{T}$ cells might explain why high-dose MnTmPyP does not enhance graft function despite the benefits of low-dose MnTmPyP in our study. In noninflammatory cells like cardiac myocytes, NOmediated apoptosis is attenuated by MnTBAP, and this is associated with changes in the proapoptotic versus antiapoptotic gene ratio of $\mathrm{Bax} / \mathrm{Bcl}-2 .^{31}$ Thus, the cell type undergoing apoptosis might play a role in determining whether the SOD mimetics are beneficial.

Proapoptotic Bcl-2 family members (eg, Bax) promote the cytosolic release of cytochrome c, whereas antiapop- totic Bcl-2 family members (eg, Bcl-2 and $\mathrm{Bcl}-\mathrm{xL}$ ) inhibit the release of cytochrome $\mathrm{c}$ and downstream activation of caspase-3. In our study there was a dosedependent inhibition of caspase-3 activation by MnTmPyP.

Collectively, these data suggest that the benefits of MnTmPyP on cardiac transplants appear to be related more to the balance of proapoptotic versus antiapoptotic gene expression rather than upstream modulation of cytokine gene expression. The effect of MnTmPyP on apoptotic signaling appears to be largely independent of TNF- $\alpha$ and FasL gene expression but rather at some downstream apoptotic signaling event.

\section{References}

1. Slakey DP, Roza AM, Pieper GM, Johnson CP, Adams MB. Delayed cardiac allograft rejection due to combined cyclosporine and antioxidant therapy. Transplantation. 1993;56:1305-9.

2. Pieper GM, Olds C, Hilton G, Lindholm PF, Adams MB, Roza AM. Antioxidant treatment inhibits activation of myocardial nuclear factor $\kappa \mathrm{B}$ and inhibits nitrosylation of myocardial heme protein in cardiac transplant rejection. Antioxid Redox Signal. 2001;3:81-8.

3. Cooper M, Lindholm P, Pieper GM, et al. Myocardial nuclear factor- $\kappa \mathrm{B}$ activity and nitric oxide production in rejecting cardiac allografts. Transplantation. 1998;66:838-44.

4. Roza AM, Pieper GM, Moore-Hilton G, Johnson CP, Adams MB. Free radicals in pancreatic and cardiac allograft rejection. Transplant Proc. 1994;26:544-5.

5. Kloc M, Mialer K, Stepowski S. Superoxide dismutase decrease in cardiac transplants. Transplantation. 1986;41:794-6.

6. Nilakantan V, Halligan NLN, Nguyen TK, et al. Posttranslational modification of manganese superoxide dismutase in acutely-rejecting cardiac transplants: role of inducible nitric oxide synthase. J Heart Lung Transplant. 2005;24:1591-9.

7. Shaw JFL, Li MKW. Free radical scavenger therapy in transplant rejection. Transplant Proc. 1987;19:1305-6.

8. Salvemini D, Wang ZQ, Zweier JL, et al. A nonpeptidyl mimic of superoxide dismutase with therapeutic activity in rats. Science. 1999; 286:304-6.

9. Vujaskovic Z, Batinic-Haberle I, Rabbani ZN, et al. A small molecular weight catalytic metalloporphyrin antioxidant with superoxide dismutase (SOD) mimetic properties protects lungs from radiationinduced injury. Free Radic Biol Med. 2002;33:857-63.

10. Cuzzocrea S, Mazzon E, Dugo L, et al. Protective effects of M40403, a superoxide dismutase mimetic, in a rodent model of colitis. Eur J Pharmacol. 2001;432:79-89.

11. Salvemini D, Mazzon E, Dugo L, et al. Pharmacological manipulation of the inflammatory cascade by the superoxide dismutase mimetic, M40403. Br J Pharmacol. 2001;132:815-27.

12. Wang W, Jittikanont $\mathrm{S}$, Falk SA, et al. Interaction among nitric oxide, reactive oxygen species, and antioxidants during endotoxemia-related acute renal failure. Am J Physiol Renal Physiol. 2003;284:F532-7.

13. Murata S, Miniati DN, Kown MH, et al. Superoxide dismutase mimetic M40401 reduces ischemia-reperfusion injury and graft coronary artery disease in rodent cardiac allografts. Transplantation. 2004;78: 1166-71.

14. Pieper GM, Nilakantan V, Zhou X, et al. Treatment with $\alpha$-phenyl$\mathrm{N}$-tert-butylnitrone, a free radical-trapping agent, abrogates inflammatory cytokine gene expression during alloimmune activation in rat cardiac allografts. J Pharmacol Exp Ther. 2005;312:774-9.

15. Shi Y, Hutchins WC, Su J, et al. Delayed cardioprotection with isoflurane: role of reactive oxygen and nitrogen. Am J Physiol Heart Circ Physiol. 2005;288:H175-84.

16. Khadour FH, Panas D, Ferdinandy P, et al. Enhanced NO and superoxide generation in dysfunctional hearts from endotoxemic rats. Am J Physiol Heart Circ Physiol. 2002;283:H1108-15. 
17. Crow JP. Manganese and iron porphyrins catalyze peroxynitrite decomposition and simultaneously increase nitration and oxidant yield: implications for their use as peroxynitrite scavengers in vivo. Arch Biochem Biophys. 1999;371:41-52.

18. Amin JK, Xiao L, Pimental DR, et al. Reactive oxygen species mediate alpha-adrenergic receptor stimulated hypertrophy in adult rat ventricular myocytes. J Mol Cell Cardiol. 2001;33:131-9.

19. Pimentel DR, Amin JK, Xiao L, et al. Reactive oxygen species mediate amplitude-dependent hypertrophic and apoptotic responses to mechanical stretch in cardiac myocytes. Circ Res. 2001;89:453-60.

20. Omar BA, Gad NM, Jordan MC, et al. Cardioprotection by Cu,Znsuperoxide dismutase is lost at high doses in the reoxygenated heart. Free Radic Biol Med. 1990;9:465-71.

21. Omar BA, McCord JM. The cardioprotective effect of Mn-superoxide dismutase is lost at high doses in the postischemic isolated rabbit heart. Free Radic Biol Med. 1990;9:473-8.

22. Piganelli JD, Flores SC, Cruz C, et al. A metalloporphyrin-based superoxide dismutase mimic inhibits adoptive transfer of autoimmune diabetes by a diabetogenic T-cell clone. Diabetes. 2002;51:347-55.

23. Zivna H, Zivna P, Navratil P, Cerny V, Holeckova M, Pliskova L. The role of cytokines and antioxidant status in graft quality prediction. Transplant Proc. 1999;31:2094.

24. Kaden J, Priesterjahn R. Increasing urinary IL-6 levels announce kidney graft rejection. Transplant Int. 2000;13(suppl):S34-41.
25. Hahn AB, Kasten-Jolly JC, Constantino DM, et al. TNF-alpha, IL-6, IFN-gamma and IL-10 gene expression polymorphisms and the IL-4 receptor alpha-chain variant Q576R: effects on renal allograft outcome. Transplantation. 2000;72:660-5.

26. Yamaura K, Ito K, Tsukioka K, et al. Suppression of acute and chronic rejection by hepatocyte growth factor in a murine model of cardiac transplantation: induction of tolerance and prevention of cardiac allograft vasculopathy. Circulation. 2004;110:1650-7.

27. Macarthur H, Couri DM, Wilken GH, et al. Modulation of serum cytokine levels by a novel superoxide dismutase mimetic, M40401, in an Escherichia coli model of septic shock: correlation with preserved circulating catecholamines. Crit Care Med. 2003;31:237-45.

28. Devadas S, Zaritskaya L, Rhee SG, Oberley L Williams MS. Discrete generation of superoxide and hydrogen peroxide by $\mathrm{T}$ cell receptor stimulation: regulation of mitogen-activated protein kinase activation and Fas ligand expression. J Exp Med. 2002;195:59-70.

29. Malassange B, Ferret PJ, Hamoud R, et al. The superoxide dismutase mimetic MnTBAP prevents Fas-induced acute liver failure in the mouse. Gastroenterology. 2001;121:1451-9.

30. Hildeman DA, Mitchell T, Kappler J, Marrack P. T Cell apoptosis and reactive oxygen species. J Clin Invest. 2003;111:571-81.

31. Arstall MA, Sawyer DB, Fukuzawa R, Kelly RA. Cytokine-mediated apoptosis in cardiac myocytes: the role of inducible nitric oxide synthase induction and peroxynitrite generation. Circ Res. 1999;85: 829-40. 